\title{
Zur Ammoniakausscheidung im Harn.
}

\author{
Von
}

Georg Landsberg, cand. med.

(Aus dem chemischen Laboratorium des pathologischen Instituts zu Berlin.)

(Der Redaction zugegangen am 18. Februar 1903.)

Vor längerer Zeit veröffentlichte ein Wiener Arzt (Dr. Emil Schwarz, Ueber den Ammoniakgehalt des Harnes, Wiener medic. Wochenschr. 1893, Nr. 3) eine Arbeit über den $\mathrm{NH}_{3}$ Gehalt des Harns, deren Resultate von den früheren Untersuchungen erheblich abwichen. Nach den Untersuchungen der verschiedensten Autoren beträgt die Tagesmenge des $\mathrm{NH}_{3}$ im Harn zwischen 0,3 und 1,2 g, im Mittel ungefähr 0,7 g. Das Resultat der erwähnten Arbeit aber war ein anderes, intlem festgestellt wurde, dass beim Versetzen des Harns mit Chloroform sofort nach seiner Entleerung die Tagesmenge des $\mathrm{NH}_{3}$ zwischen 0 und $0,432 \mathrm{~g}$, im Mittel $0,155 \mathrm{~g}$ betrug. Die grösseren $\mathrm{NH}_{3}$-Werthe der früheren Autoren sollten dadurch eine Erklärung finden, dass eine theilweise Zersetzung des Harnstoffs im Harn eingetreten war; eine Zersetzung, die durch den Ghloroformzusatz hier vermieden war.

Diese Angaben einer Nachprüfung zu unterziehen, der sie meines Wissens bisher nicht unterzogen worden sind, erschien schon deshalb geboten, weil das Ammoniak bisher als ein constanter Bestandtheil des menschlichen Harns gilt, während nach den Angaben der fraglichen Arbeit bei einer Zahl von 17 Untersuchungen dreimal nicht einmal Spuren von Ammoniak im Urin nachzuweisen waren. Die Untersuchungen wurden in folgender Weise ausgeführt. Es wurde bei einer 
Entleerung der Blase ein Theil des Urins in ein Chloroform enthaltendes Gefäss gelassen, während der andere Theil ohne Chloroformzusatz blieb. Die Behandlung beider Portionen, die stets ganz gleichmässig war, ergibt sich aus folgender Tabelle:

\begin{tabular}{|c|c|c|c|c|c|c|c|c|c|c|}
\hline $\begin{array}{l}\text { Zur } \\
\text { Bestimmung } \\
\text { verwendete } \\
\text { Urinmenge }\end{array}$ & $\begin{array}{l}\text { Stchen } \\
\text { des } \\
\text { Urins } \\
\text { vor } \\
\text { Beginn } \\
\text { der } \\
\text { Bestim- } \\
\text { mung }\end{array}$ & $\begin{array}{c}\text { Anzahl } \\
\text { der Tage } \\
\text { des } \\
\text { Stehens } \\
\text { im } \\
\text { S chlöss- } \\
\text { ing 'schen } \\
\text { Apparat }\end{array}$ & $\begin{array}{l}\text { Vorgelegte } \\
\text { Normal- } \\
\text { säure }\end{array}$ & $\begin{array}{c}\text { Ge- } \\
\text { bunden } \\
\text { in der } \\
\text { Harn- } \\
\text { portion } \\
\text { mit } \\
\mathrm{CHCl}_{3}\end{array}$ & $\begin{array}{l}\text { Ge- } \\
\text { buuden } \\
\text { in der } \\
\text { Harn- } \\
\text { portion } \\
\text { ohne } \\
\mathrm{CHCl}_{3}\end{array}$ & \multicolumn{5}{|c|}{$\begin{array}{c}\text { Differenz } \\
\text { der Bindung in beiden } \\
\text { Portionen }\end{array}$} \\
\hline 1. $50(\mathrm{~cm} 1)$ & $0 \mathrm{Tag}$ & 3 & $10 \mathrm{ccm} \mathrm{n} / 4$ & 5,8 & 6,1 & $-0,3 \mathrm{n} /$ & 1/4-Så̃ & ore in & Por & mit $\mathrm{CHO}_{3}$ \\
\hline 2. 50 & 0 & 3 & $10 \curvearrowright$ & 5,7 & 5,6 & $+0,1$ & $\gg$ & 》 & 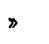 & , \\
\hline 3. $50 »$ & 0 & 3 & $10 \gg$ & 5,2 & 5,1 & $+0,1$ & $\gg$ & $»$ & $\gg$ & $\gg$ \\
\hline 4. 50 » & 0 & 2 & $25 \gg n / 10$ & 15,6 & 14,9 & $-0,7 \mathrm{n} /$ & $1 / 10$ & $\gg$ & $\gg$ & > \\
\hline 5. 50 & 1 & 3 & $25 \gg$ & 14,1 & 13,9 & $+0,2$ & $»$ & $»$ & $\triangleright$ & $\triangleright$ \\
\hline 6. 50 & 1 & 2 & $25 \gg \gg$ & 11,2 & 10,6 & $+0,6$ & $\gg$ & 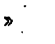 & » & 8 \\
\hline 7. $50 »$ & 1 & 4 & $25 》$ & 14,3 & 14,3 & 0,0 & $»$ & & $»$ & $\triangleright$ \\
\hline 8. 50 & 1 & 3 & $25 \gg$ & 10,0 & 10,4 & $-0,4$ & $D$ & 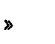 & \#. & $\gg$ \\
\hline 9. $50 \gg$ & 1 & 4 & $25 \gg$ & 11,6 & 10,8 & $+0,8$ & 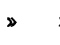 & 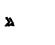 & 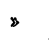 & $>$ \\
\hline 10. $50 »$ & 1 & 3 & $25 \gg>$ & 12,4 & 12,6 & $-0,2$ & 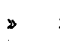 & $»$ & $>$ & $\triangleright$ \\
\hline 1.1. $50 »$ & $1 》$ & 3 & $25 \gg$ & 13,7 & 13,5 & $+0,2$ & $»$ & $\triangleright$ & $\gg$ & $>2$ \\
\hline
\end{tabular}

Aus dieser Tabelle geht hervor, dass bei den elf vorgenommenen Bestimmungen sechsmal in der mit $\mathrm{CHCl}_{3}$ versetzten Portion etwas mehr Säure gebunden war, viermal in der ohne $\mathrm{CHCl}_{3}$ angesetzten, während einmal die Bindung in beiden Portionen die gleiche war: Die Differenzen betrugen, auf n/10-Säure bezogen, zwischen 0,0 und $0,8 \mathrm{ccm}$ und dürften zum Theil auf die Fehlerquellen beim Ansetzen und Titriren zurückzuführen sein, zum Theil vielleicht auch darauf, dass der Urin in der Blase selbst nach Kọ̈perbewegung. nicht vollständig gleich zusammengesetzt ist. Jedenfalls ergeben die vergleichenden Bestimmungen, dass von einer Zersetzung des nicht conservirten Harns nicht die Rede sein kann.

1) Absichtlich wurde die Harnmenge so gross gewählt, um für den Fall, dass der sofort in Chloroform entleerte Harn nur Spuren ron Ammon enthalten sollte, auch diese. nicht zu übersehen. 
Um ferner festzustellen, wie hoch der $\mathrm{NH}_{3}$-Gehalt in der Tagesmenge war, wurde der Harn bei acht Bestimmungen stets in Chloroform enthaltende Gefässe gelassen und stets stark mit dem Chloroform durchschüttelt. Die acht Bestimmungen ergaben:

\begin{tabular}{|c|c|c|c|}
\hline Tagesmenge & $\begin{array}{c}\text { Zur } \\
\text { Bestimmung } \\
\text { im } \\
\text { Schlössing- } \\
\text { schen Apparat } \\
\text { verwendete } \\
\text { Menge }\end{array}$ & $\begin{array}{c}\text { Menge } \\
\text { der } \\
\text { gebundenen } \\
\text { n/10-Säure in } \\
\text { Cubik- } \\
\text { centimetern }\end{array}$ & $\mathrm{NH}_{3}-\mathrm{T}$ agesmenge \\
\hline 1. 1100 & $100 \mathrm{ccm}$ & 33,9 & $0,634 \mathrm{~g}$ \\
\hline 2. 1180 & 100 & 22,0 & $0,44.1$, \\
\hline 3. 1030 & 100 & 34,4 & 0,601 \\
\hline 4. 1330 & 100 & 21,2 & 0,478 \\
\hline 5. 1820 & 100 & 22,0 & 0,681 \\
\hline 6. 1150 & 100 & 26,5 & 0,518 \\
\hline 7. 1190 & 100 & 23,9 & 0,484 \\
\hline 8. 1250 & 100 & 35,6 & 0,757 》 \\
\hline
\end{tabular}

Die $\mathrm{NH}_{3}$-Tagesmenge schwankte also zwischen $0,441 \mathrm{~g}$ und $0,757 \mathrm{~g}$ und betrug im Mittel $0,574 \mathrm{~g}$. Alle gefundenen Werthe lagen innerhalb der bisher als normal angesehenen.

Die Angaben der nachgeprüften Arbeit haben sich demnach als unhaltbar erwiesen. Ob die $\mathrm{zu}$ geringen Werthe derselben der Bestimmungsmethode zuzuschreiben sind, die im Abdestilliren des mit Kalkmilch versetzten Harns bei $30-50^{\circ}$ ins Vacuum bestand, oder aber den geringen zur Bestimmung verwandten Urinmengen (nur $5 \mathrm{ccm}$ in einigen Fällen), muss dahingestellt bleiben. Thatsache ist, dass der $\mathrm{NH}_{3}$-Gehalt des Harns auch nach Chloroformconservirung keine wesentlichen Unterschiede von dem des nicht conservirten Harns darbietet, dass er mithin sicher nicht auf theilweiser Zersetzung des Harnstoffs ausserhalb des Körpers beruht. 